\section{Line Pattern Retrieval Using Relational Histograms}

\author{
Benoit Huet, Member, IEEE Computer Society, \\ and Edwin R. Hancock
}

\begin{abstract}
This paper presents a new compact shape representation for retrieving line-patterns from large databases. The basic idea is to exploit both geometric attributes and structural information to construct a shape histogram. We realize this goal by computing the $\mathrm{N}$-nearest neighbor graph for the lines-segments for each pattern. The edges of the neighborhood graphs are used to gate contributions to a two-dimensional pairwise geometric histogram. Shapes are indexed by searching for the line-pattern that maximizes the cross correlation of the normalized histogram bin-contents. We evaluate the new method on a database containing over 2,500 line-patterns each composed of hundreds of lines.
\end{abstract}

Index Terms-Image database, line patterns, content-based retrieval, relational representation, geometric features, histogram comparison.

\section{INTRODUCTION}

LINE patterns have proved to be highly effective for shape representation and recognition [1], [2]. Broadly speaking there are two sources of information that can be tapped for line-pattern recognition. The first of these are the intrinsic geometric attributes of the line-segments, namely their angles and lengths. The second source of information is structural, and is provided by the relational arrangement of the lines. Although both structural indexing [3], [4], [5] and attribute histograms [3], [4] have been separately used to recall image data from databases, they have not been used in conjunction. The aim in this paper is to fill this gap in the literature by presenting a compact line pattern representation that can be used for rapid object retrieval from large databases.

The idea is a simple one. We construct a histogram of Euclidean invariant geometric attributes for line-pairs connected by the edges of a nearest-neighbor graph. Recognition is effected by comparing the normalized histograms in a database of line patterns with the histogram for the query image or model. Our measure of histogram similarity is the Bhattacharyya distance. It is the idea of using a nearest neighbor graph to localize the features used in the pairwise geometric histogram which represents the main contribution in this paper.

There are a number of related ideas reported in the literature. It is the idea of using a neighborhood graph to gate histogram entries together with our aim of indexing into large databases, which gives us a novel edge over the work of Thacker et al. [6] on pairwise geometric histograms. Moreover, our method is scale-invariant and we reduce the computational overheads by conglomerating the line segments in a single histogram rather than having a histogram per line-segment. Viewed from the perspective of structural hashing [7] [8], our method uses a compact histogram representation rather than a large hash-table which can grow to unmanageable size for complex scenes. The use of local geometric features is common to a number of object recognition strategies. For instance, geometric hashing attempts to tabulate local features in such a way as to index object-shape [9], [10]. Stein and Medioni [1] provide one of the most ambitious hashing schemes which

- The authors are with the Department of Computer Science, University of York, Y10 5DD, United Kingdom. E-mail: \{huetb, erh\}@cs.york.ac.uk.

Manuscript received 7 Apr. 1998; revised 19 Oct. 1999.

Recommended for acceptance by Y.-F. Wang.

For information on obtaining reprints of this article, please send e-mail to: tpami@computer.org, and reference IEEECS Log Number 107654. draws on relatively complex polygonal supersegments as the geometric primitives. Although this representation is considerably more sophisticated than our use of simple-line segment pairs, it is potentially more fragile as a result of sensitivity to the token grouping process. Several methods use neighborhood graphs or more complex relational structures for feature-matching [11], [12], [13]. However, these methods require the identification of a set of global token correspondences. In our method there is no such requirement since we compare objects at the histogram level without having to perform detailed alignment or correspondence. It is this feature that also distinguishes our work from that of Grimson and Lozano-Perez [12] who use pairwise relational constraints to identify objects via the search of the interpretation tree.

\section{Pairwise Geometric Attributes}

Our overall goal is to retrieve line-patterns in a rotation, translation, and scale-invariant manner. We offer two Euclidean invariant pairwise geometric attributes aimed at enhancing recognition performance. Suppose that we denote the line segments indexed $(a b)$ and $(c d)$ by the vectors $\underline{\mathbf{x}}_{a b}$ and $\underline{\mathbf{x}}_{c d}$, respectively. The vectors are directed away from their point of intersection. The relative angle attribute is given by

$$
\alpha_{a b, c d}=\arccos \left[\frac{\underline{\mathbf{x}}_{a b} \cdot \underline{\mathbf{x}}_{c d}}{\left|\underline{\mathbf{x}}_{a b}\right|\left|\underline{\mathbf{x}}_{c d}\right|}\right] ;
$$

From the relative angle, we compute the directed relative angle. This is an extension to the attribute used by Evans et al. [14], that consists of giving the relative angle a positive sign if the direction of the angle from the baseline $\underline{\mathbf{x}}_{a b}$ to its partner $\underline{\mathbf{x}}_{c d}$ is clockwise and a negative sign if it is counterclockwise. This allows us to extend the range of angles describing pairs of segments from $[0, \pi]$ to $[-\pi,-\pi]$ and, therefore, reduce retrieval errors associated with angular ambiguities.

Our requirements of the length based attribute are twofold. In the first instance, we would like a scale-invariant measure. For this reason, we make use of the normalized length ratio between the oriented baseline vector $\underline{\mathbf{x}}_{a b}$ and the vector $\underline{\mathbf{x}}_{i b}$ joining the end $(b)$ of the segment $(a b)$ to the intersection of the segment $(c d)$. The second requirement, is to provide additional discrimination when lines are parallel to one another. For this reason, the length ratio attribute used in our histograms is

$$
\vartheta_{a b, c d}=\frac{1}{\frac{1}{2}+\frac{D_{i b}}{D_{a b}}} .
$$

The physical meaning of this attribute deserves further comment. The minimum value of $D_{i b}$ occurs when the line $c d$ intersects the line $a b$ at its midpoint (see Fig. 1). As a result, $D_{i b} \geq \frac{1}{2} D_{a b}$ and, hence, $\frac{D_{i b}}{D_{a b}} \geq \frac{1}{2}$. The physical range of the attribute $\vartheta_{a b, c d}$ is, therefore, $(0,1]$. A value of 0 indicates that the two segments are parallel, while a value of 1 indicates that the two segments intersect at the middle point of the baseline.

Both the directed relative angle and the length ratio attributes are invariant to changes of scale, rotation, and translation. This is an important advantage over the representation proposed by Evans et al. [14], if the technique is to be used for shape retrieval from real world image databases, where the size of objects, or, more generally shapes, is not known in advance.

\section{Histogram Representation}

The novel idea introduced in this paper is to gate the entries in the pairwise geometric histogram using the edge-set of the neighborhood graph. Although our histograms integrate information over 


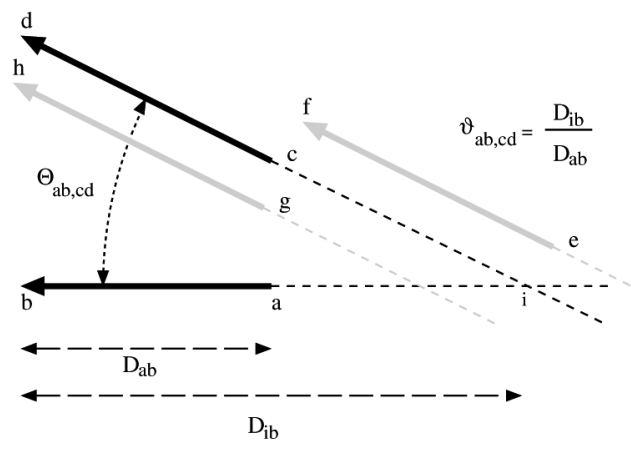

Fig. 1. Geometry used for pairwise shape representation.

the complete line-pattern, contributions are gated by the local structure conveyed by a neighborhood graph. The motivation here is that local object representations are more robust to occlusion, missing or extra features, and noise. Moreover, by integrating both geometric attributes and structural information into a single histogram, we provide a compact relational representation of line-structure. This gating process greatly reduces the number of entries in each histogram, hence, diminishing the risk of histogram saturation. Structural gating ensures that this representation does not become saturated. As pointed out by Stricker and Swain [15], this feature can severely restrict the recall capacity of the recognition process.

Our idea contrasts with a number of related contributions in the literature. For instance, Evans et al. [14] effectively have a local representation of line-pattern structure which is less compact than ours since it employs one histogram per line-segment. Moreover, their attributes are not scale invariant. Di Mauro et al. [16] idea of using a region-of-interest is close to that of using a neighborhood graph. However, their regions are controlled by a scale parameter. By virtue of their structural character, neighborhood graphs are scale-invariant.

Our structural representation of the line patterns is a N-nearest neighbor graph for the center points of the line-segments. Suppose that the node set of the nearest neighbor graph, i.e., the centers of

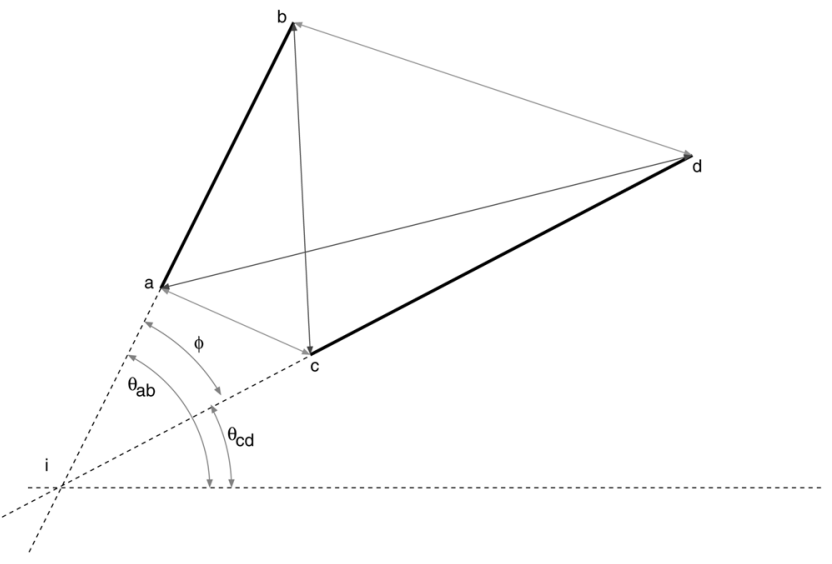

Fig. 2. Computing the pairwise geometric attributes from line segments $(a b)$ and $(c d)$.

the line segments extracted from an image, is denoted by the set $V$. The edges of the graph indicate that the Euclidean distances between pairs of line center points satisfy the N-nearest neighbor condition. The edge-set of the nearest neighbor graph is $E \subset V \times V$. The set of edges is used to construct the structurally gated geometric histogram. The bin-incrementing process can be formally described as follows: Let $i$ and $j$ be two segments extracted from the raw image. The angle and length ratio attributes $\alpha_{i j}$ and $\vartheta_{i j}$ are binned provided the two segments are connected by an edge i.e., $(i, j) \in E$. If this condition is met, then the bin $H(I, J)$ spanning the two attributes is incremented as follows:

$$
H(I, J)= \begin{cases}H(I, J)+1 & \text { if }(i, j) \in E \text { and } \alpha_{i, j} \in A_{I} \text { and } \vartheta_{i, j} \in R_{J} \\ H(I, J) & \text { otherwise, }\end{cases}
$$

where $A_{I}$ is the range of directed relative angle attributes spanned by the $I$ th horizontal histogram-bin and $R_{J}$ is the range of length ratio attribute spanned by the $J$ th vertical histogram bin. Each histogram contains $n_{A}$ relative angle bins and $n_{R}$ length ratio bins. The normalized gated geometric histogram bin-entries are computed as follows:

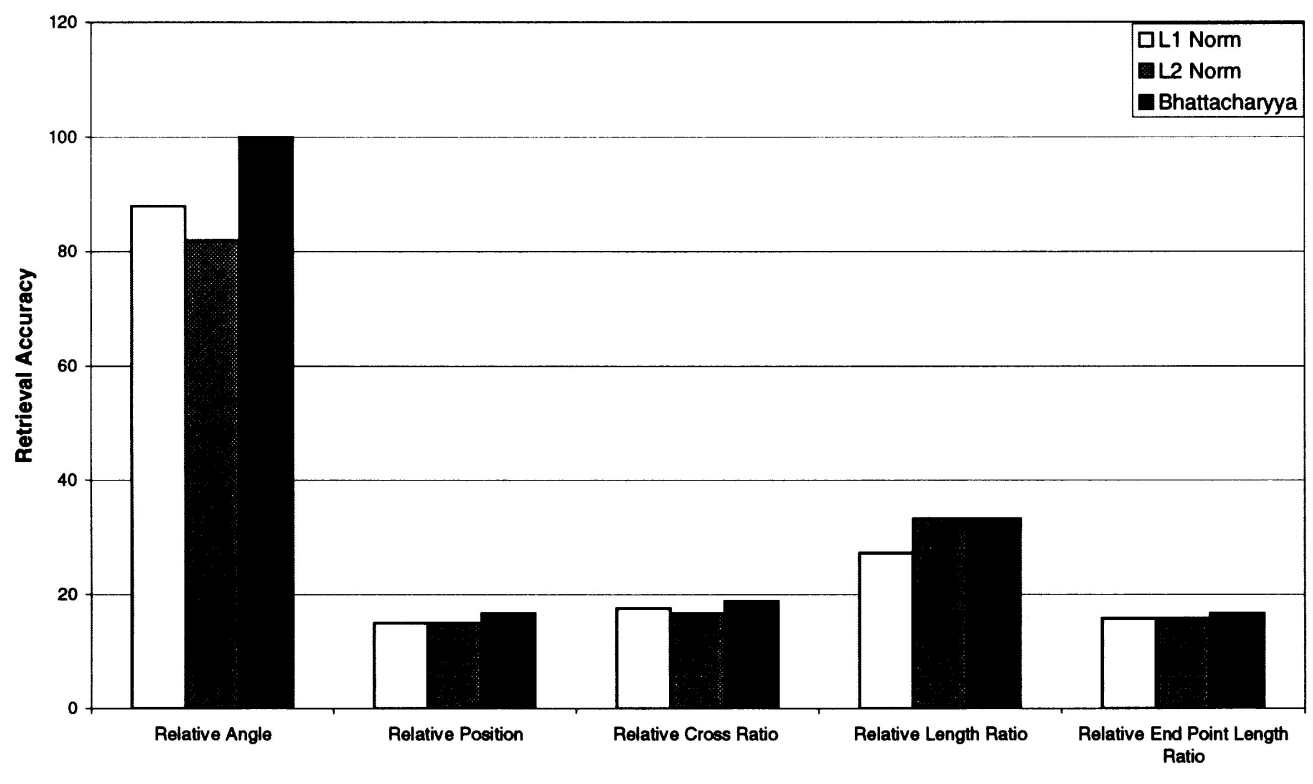

Fig. 3. Performance results using various attributes for shape similarity retrieval. 


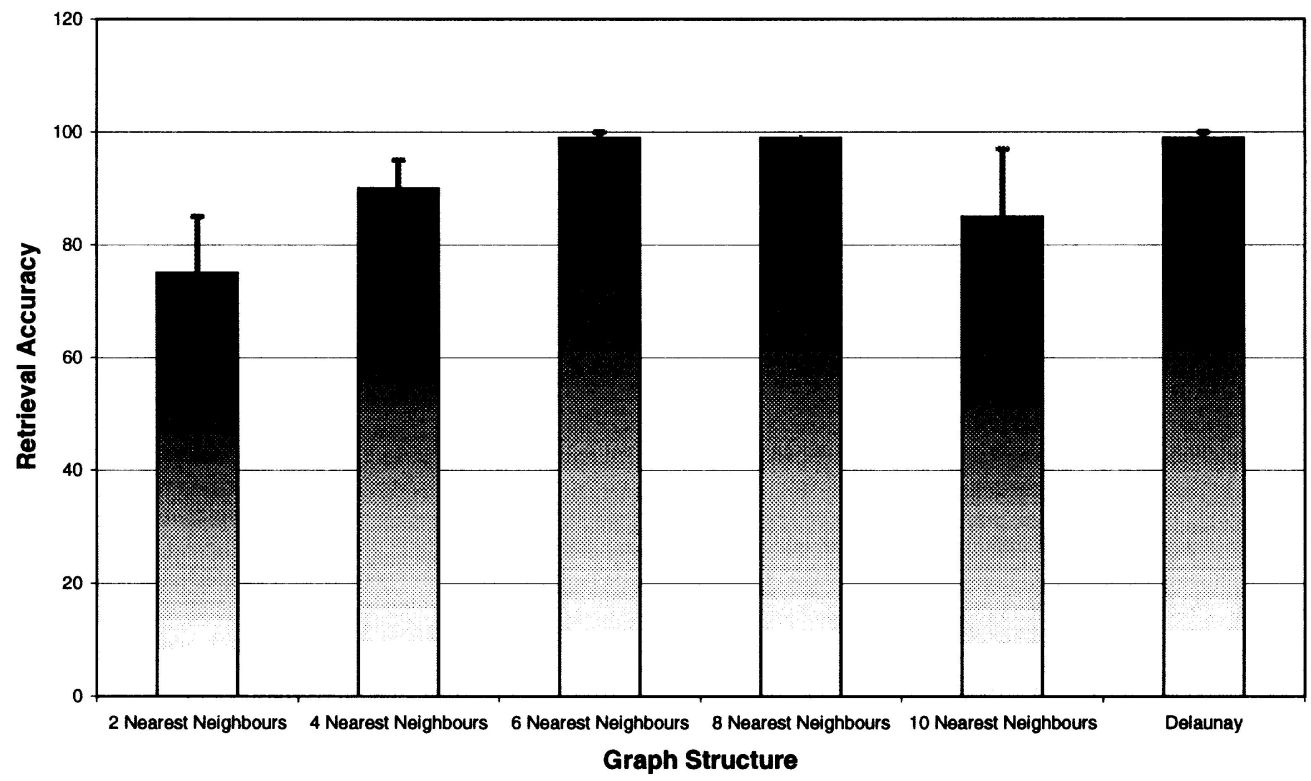

Fig. 4. Effect of the choice of relational structure on retrieval accuracy.

$$
h(I, J)=\frac{H(I, J)}{\sum_{I^{\prime}=1}^{n_{A}} \sum_{J^{\prime}=1}^{n_{R}} H\left(I^{\prime}, J^{\prime}\right)} .
$$

The gating process greatly reduces the number of entries in each histogram, hence, diminishing the risk of histogram saturation. As an illustrative example, some of the images used in our experiments contain over 1,000 segments. Such a linepattern would result in over $(1,000 *(1,000-1)) / 2)=498,500$ histogram entries. By contrast, in our structurally gated histograms, based on a six nearest neighbor graph, there are on average only $1,000 * 6=6,000$ entries.

The histogram granularity is as follows: The 36 vertical bins of our histogram represent the directed relative angle varying from $-\pi$ to $\pi$. The 12 horizontal bins represent the length ratio attribute ranging from 0.5 to $\infty$. We use a nonuniform binning for this attribute to take account of three specific cases of line-geometry. The leftmost bin, is reserved for line-segment pairs which intersect each other within the baseline. The second bin, is reserved for linesegment pairs that intersect at the origin of the baseline segment. These line-pairs are potential junction candidates and convey important perceptual cues. The third reserved bin, is rightmost in the histogram and contains parallel lines. The nine remaining horizontal bins uniformly represent line-segment pairs for which the intersection is outside the baseline.

Line patterns are retrieved from the database on the basis of minimum Bhattacharyya distance to the query pattern. Suppose that $h_{Q}$ is the normalized histogram for a query line-pattern and $h_{D}$ represents the corresponding normalized histogram contents of one of the histograms contained within the database. The Bhattacharyya distance between the two histograms is equal to

$$
B(Q, M)=-\ln \sum_{I=1}^{n_{A}} \sum_{J=1}^{n_{R}} \sqrt{h_{Q}(I, J) \times h_{D}(I, J)} .
$$

The Bhattacharyya distance uses the correlation between the bin contents as a measure of pattern similarity. This means that bins of zero contents do not contribute to the distance. In other words, the measure favors model-data associations which are dominated large-contents bins. For highly structured histograms, i.e., those which are not uniformly populated, this can lead to the selection of

matches in which there is a strong congruency between the salient structure of model and data.

We acknowledge there are more sophisticated ways of comparing histogram bin-contents. For instance, the recently reported earth-movers distance of Rubner et al. [17] computes histogram distance by allowing for leakage between adjacent bins. However, this is an iterative distance computation. It is, hence, more computationally expensive than our distance measure.

\section{Algorithm Optimization}

We commence our experimental study by providing some empirical results, which point to the best choice of geometric attributes and order of relational structure.

\subsection{Pairwise Geometric Attributes}

We compare the retrieval performance obtained by using the pairwise attributes described in Section 2 with those described by Bray and Hlavac [18]. Suppose that the line with endpoints indexed $(a b)$ has length $l_{a b}$ and orientation $\theta_{a b}$ as shown in Fig. 2. The alternative attributes are defined as follows:

1. the relative orientation between the lines indexed $(a b)$ and $(c d) \phi_{a b, c d}=\min \left[\left(\theta_{a b}-\theta_{c d}\right),\left(\alpha_{c d}-\alpha_{a b}\right)\right]$;

2. the ratio of line-segment length

$$
R_{a b, c d}=\frac{\min \left[l_{a b}, l_{c d}\right]}{\max \left[l_{a b}, l_{c d}\right]}
$$

3. the ratio of segment end-point distances

$$
E_{a b, c d}=\frac{\min \left[l_{a c}, l_{b d}\right]}{\max \left[l_{a c}, l_{b d}\right]}
$$

4. the line-segment projection cross-ratio

$$
X_{a b, c d}=\frac{\min \left[l_{a d}, l_{b c}\right]}{\max \left[l_{a d}, l_{b c}\right]} .
$$




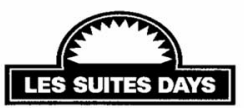

(a)

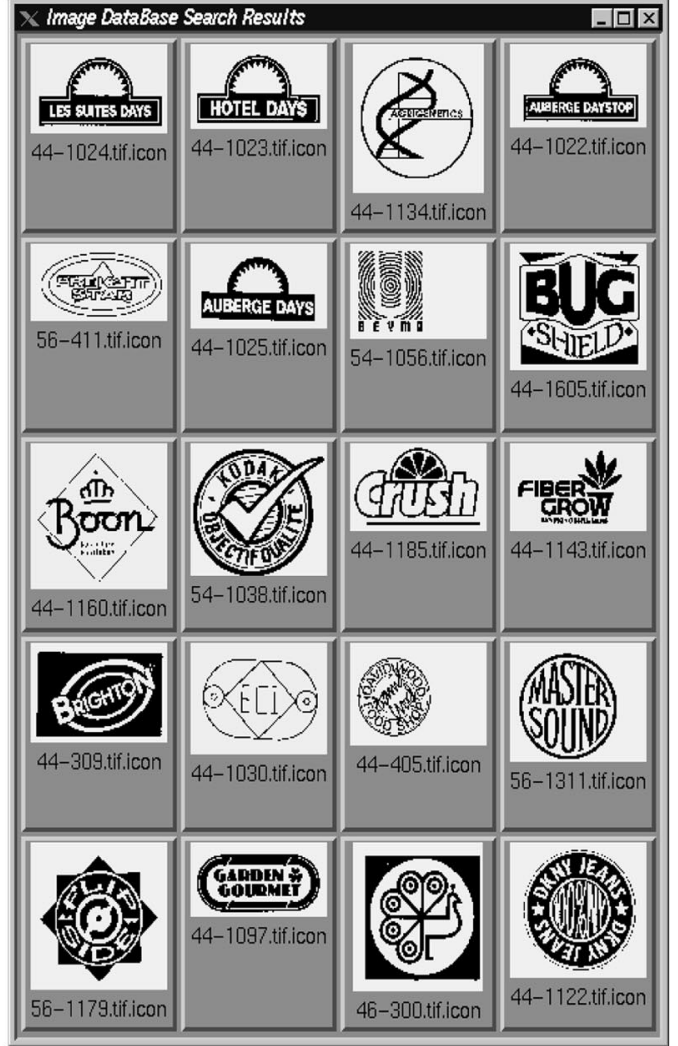

(b)

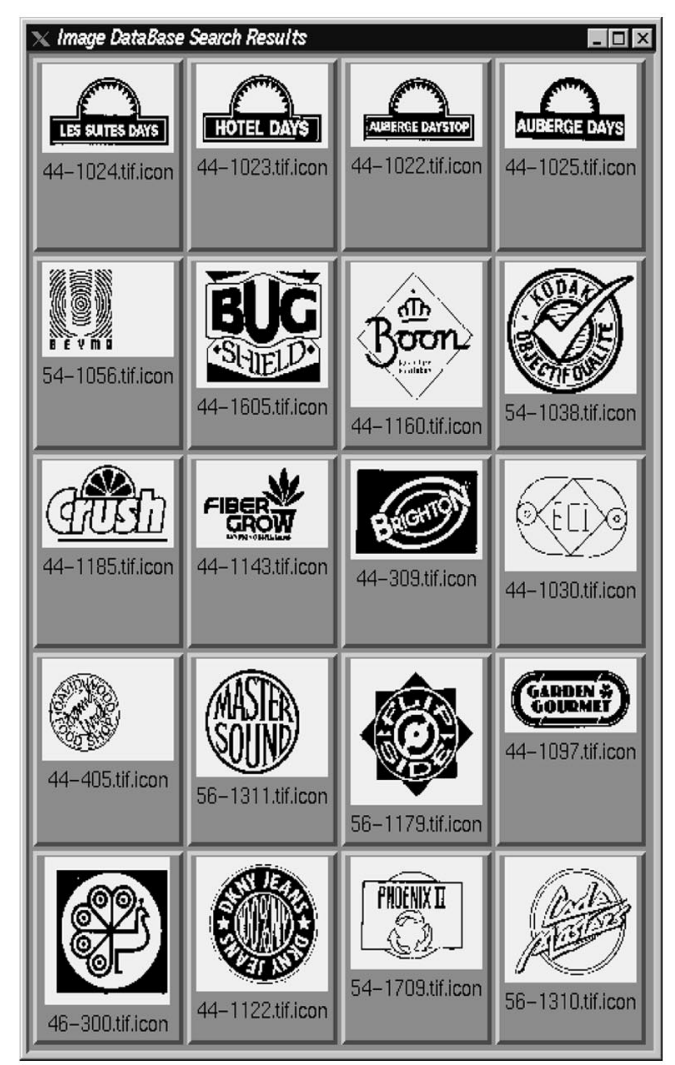

(c)

Fig. 5. The results of querying the database with the "Le Suites Days" logo: (a) The images are ordered from left-to-right and top-to-bottom in increasing distance from the query image, which is shown at the top of the figure. (b) The left-hand panel is the result obtained with the ungated histogram. (c) The right-hand panel is the result obtained when a relational histogram is used.

Fig. 3 shows the retrieval accuracy obtained using each of these attributes and compares them with those described in Section 2. In each case we measure the histogram distance using the L1 norm, L2 norm and Bhattacharyya distance. The variation in performance accuracy due to the different attributes is much greater than the variation due to choice of distance measure. It is clear that the relative angle attribute offers the best retrieval accuracy. By contrast, each of the length ratio attributes emerge as performing relatively poorly as a result of fragility under segmentation error. However, it is the relative length ratio $\vartheta$ that delivers the best performance. Based on this experiment, we select the directed relative angle attribute and the directed relative length attribute described in Section 2 as the two attributes to be used in our histogram representation.

\subsection{Graph Order}

In this subsection, we consider the role of relational structure on recall performance. Fig. 4 compares the retrieval accuracy achieved with a number of N-nearest neighbor graphs and the Delaunay graph. The best performance is obtained with the Delaunay graph and the N-nearest neighbor graphs of order 6 to 8 . This is an observation of critical importance for our study of line-pattern indexation. The computation of the Delaunay graph is time consuming. It is, therefore, not particularly well suited for large image databases. We will, therefore, use the six nearest neighbor graph. This offers the best compromise between efficiency of computation and retrieval accuracy.

\section{RECOGNITION EXPERIMENTS}

In this section, we present some examples to illustrate the effectiveness of the relational histogram for line-pattern retrieval. The database used for our experiments consists of line patterns extracted from over 2,500 trademarks and logos. Concealed in the database are the line segments extracted from 15 aerial infrared line scan images. The dominant line-features in these images are road networks. Two of the aerial images contain line structure that corresponds with a digital map. We will use this map as an inexact query into the database since it has significant differences in both structure and geometry. We compare the results with those obtained with the standard ungated geometric histogram. The results are presented in the form of panels of "thumbnail" images from the database used in our retrieval experiments. The "thumbnails" are ordered (from left-to-right and top-to-bottom), according to their distance from the query image.

Our first example uses the "Le Suites Days" logo. The recognition results are shown in Fig. 5. There are four logos in the database that have the same shape, but which carry a different legend (Hotel Days, Auberge Daystop, and Auberge Days). In the 


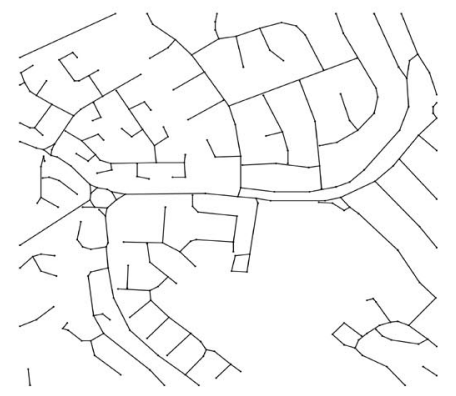

(a)

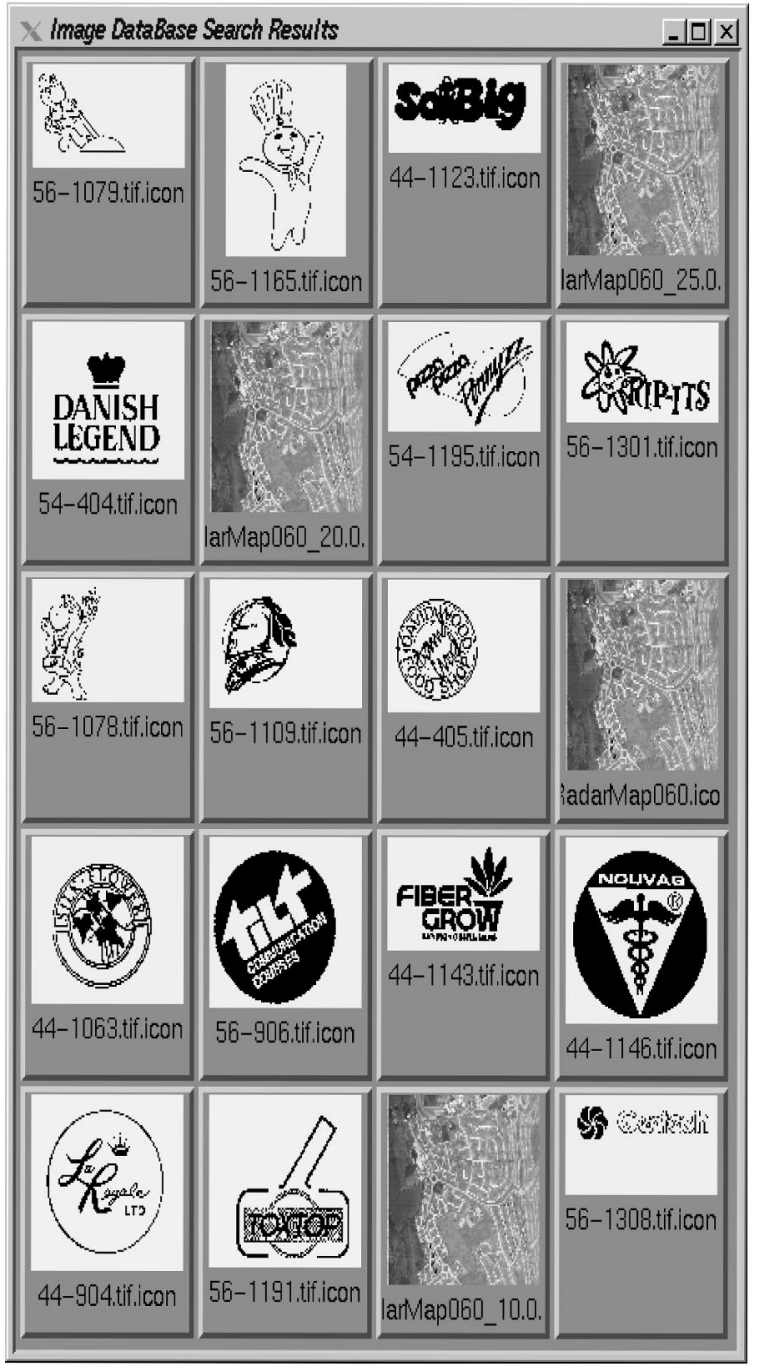

(b)

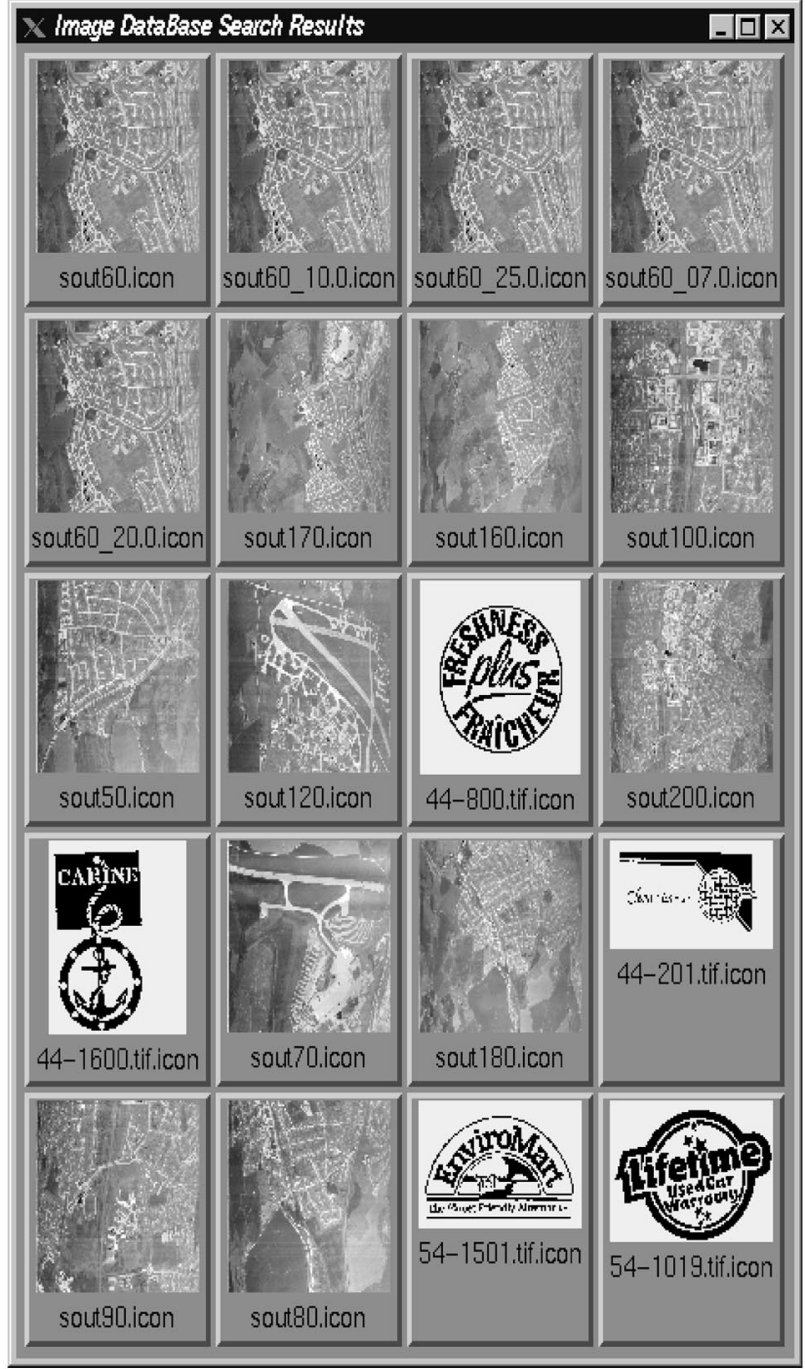

(c)

Fig. 6. The result of querying the database with the digital map: (a) The images are ordered from left-to-right and top-to-bottom in increasing distance from the query image, which is shown at the top of the figure. (b) The left hand panel is the result obtained with the ungated histogram. (c) The right hand panel is the result obtained when a relational histogram is used.

case of both the relationally gated histogram and its ungated counterpart, the target logo is correctly retrieved in the top ranked position. However, in the case of the relational histogram, the three logos with the same shape but different legends are ranked from second to fourth. In the ungated case, these three logos are more dispersed and appear at rank positions 2, 4, and 6 .
Having demonstrated our retrieval scheme on an exact query, we now turn to the more complex example on a similarity query. In this case, our example is furnished by the cartographic section of the database. The line-patterns contained in the cartographic section of the database are segmented from infrared line-scan images of urban and semiurban areas. The Digital Map (or 


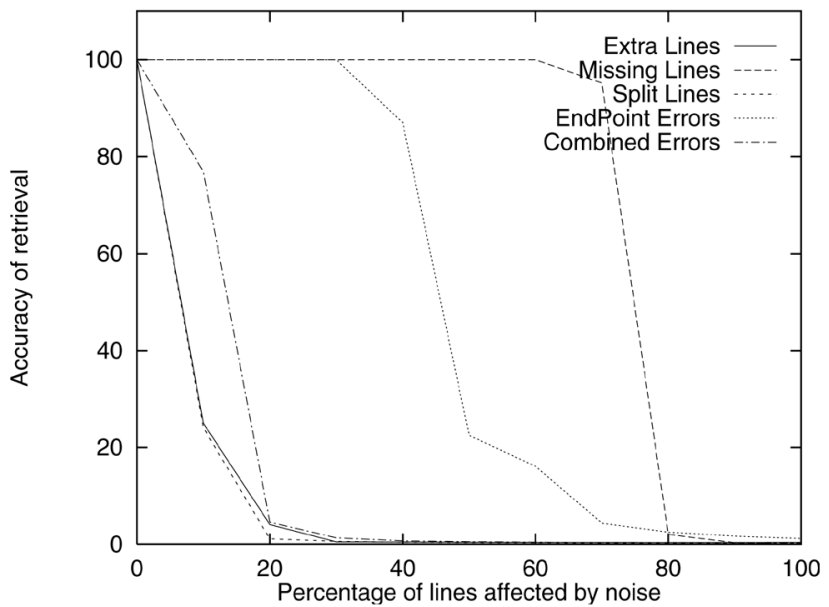

(a)

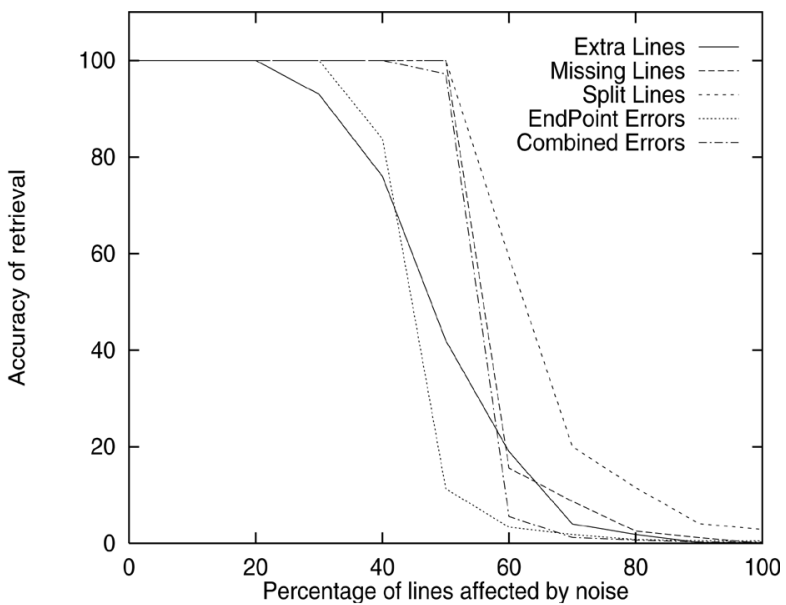

(b)

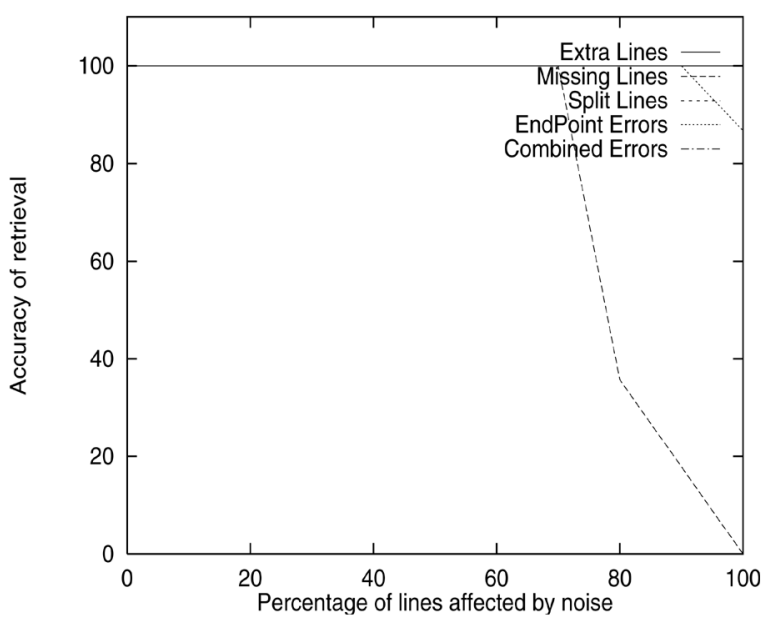

(c)

Fig. 7. Effect of various kinds of noise to the retrieval performance when the target and the query are the same apart from added noise. (a) Ungated Histogram. (b) Relational Histogram. (c) Graph Matching.

cartographic model) used to query the database represents a road network known to appear in a subset of the images contained within the database. The two images labeled Map060 and Map170 both contain the road network area depicted in the cartographic model, but are acquired when the imaging apparatus is making different altitude passes over the scene.

The infrared line-scan process produces significant barrel distortion. This is due to the rotating mirror optics underlying used to sample the infrared images. Since the digital map is cartographic in origin and the line-patterns are segmented from the infrared, there are also significant structural differences. As a result, the digital map can be regarded as a similarity query. We have also introduced five over- and under-segmented sets of line patterns into the database to investigate the effect of segmentation error on retrieval performance. Ideally, the result of a database query using the Digital Map depicted in Fig. 7 is a retrieval where both the various line-segmentations of the images Map060 and Map170 are recalled with the highest ranks.

The ranked "thumbnail" images are compared for the relationally gated and ungated histograms in Fig. 6. In the case of the relational histograms, the five different segmentations of the target image (Map060) are top-ranked (i.e., Map060_10, etc.). The result of controlled over and under segmentation of the image results in significant variation in the number of segments used to construct the histogram representation. The retrieval is not specifically affected by these segmentation systematics as can be seen in Fig. 6 . The high altitude image of the same area (i.e., Map170) appears at rank 6.

In the case of the ungated histogram the different segmentations of the target image are badly dispersed. Four of the five different segmentations appear at rank positions 4, 6, 12, and 19. In other words, the retrieval performance is very poor.

\section{Recognition Sensitivity to Segmentation ERRORS}

The aims in this section are twofold. First, we investigate the sensitivity of the gated relational histogram, to the systematics of the line-segmentation process. Second, we aim to compare the performance with that obtained using both the simpler ungated histogram and a more sophisticated graph-matching technique. The graph-matching algorithm [19] is a simplification of the 


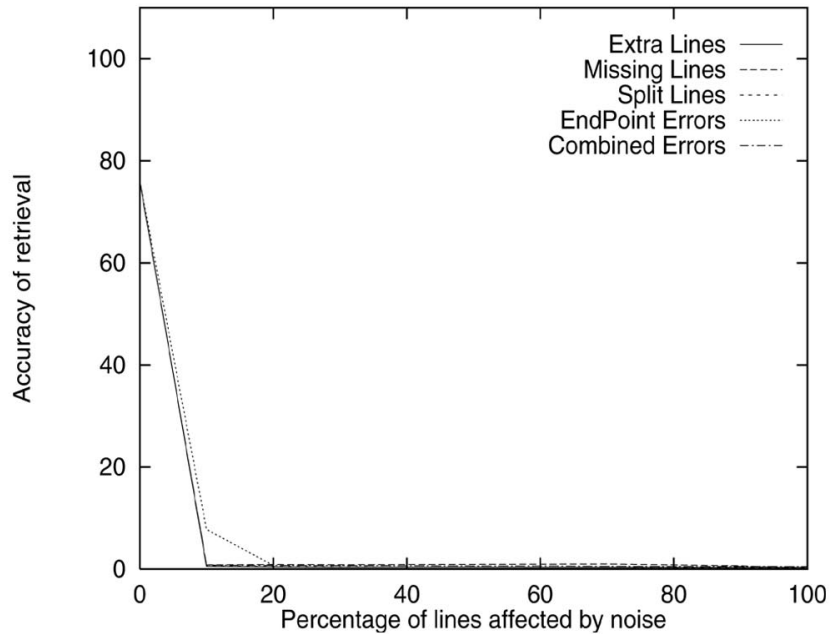

(a)

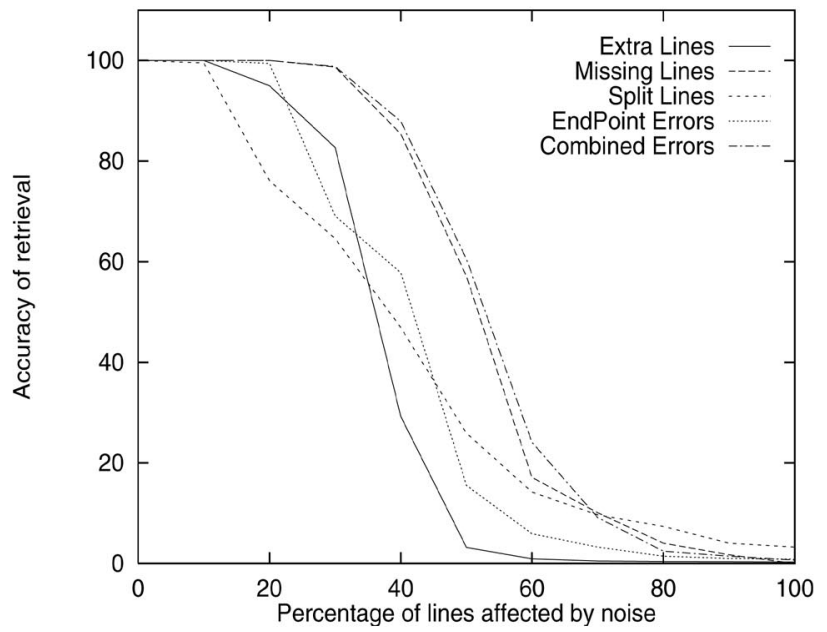

(b)

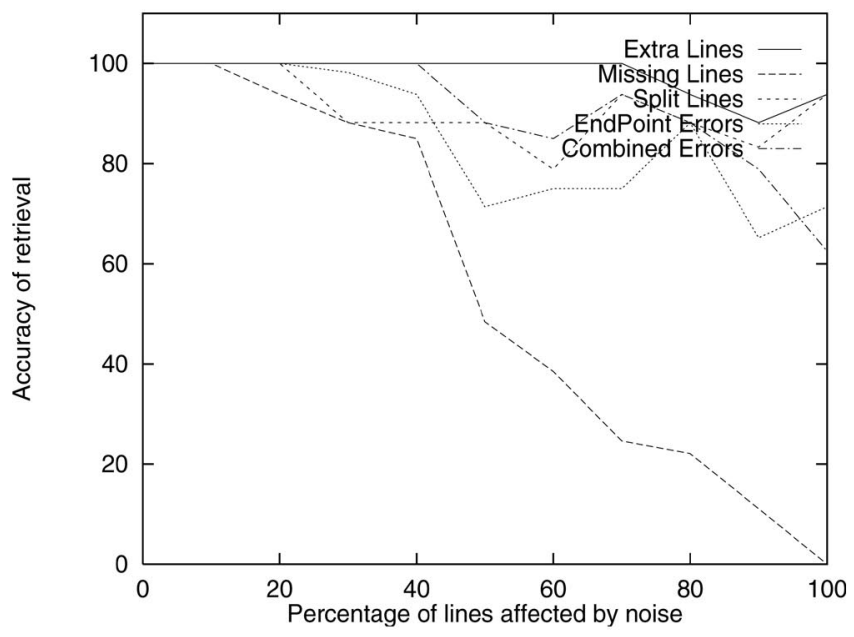

(c)

Fig. 8. Effect of various kinds of noise to the retrieval performance when the targets and the query are similar. (a) Ungated Histogram. (b) Relational Histograms. (c) Graph matching.

discrete relaxation method recently reported by Wilson and Hancock [13]. It uses edge-consistency to find a set of explicit correspondence matches between the line tokens in the query and database patterns. The correspondence is used to rank the graphs in the database according to their relative consistency. The measure of relative consistency is the fraction of edges in the database graph that are consistently matched onto the query graph.

To these ends, we have simulated the segmentation errors that can occur when line-segments are extracted from realistic image data. Specifically, the different error processes that we have investigated are listed below:

- Extra lines: Here, we have added additional lines at random locations. The lengths and angles of the added lines have been generated by randomly sampling the distribution for the existing image-segments.

- Missing lines: Here, we have deleted a known fraction of line-segments at random locations.
- Split lines: Here, a predefined fraction of lines have been split into two segments. The splitting process is effected by deleting an internal fraction of each line-segment. The deleted segment is randomly positioned along the line. The fraction of the line deleted is uniformly sampled from the range $(0,1)$.

- Segment end-point errors: Here, we have introduced random displacements in the end-point positions for a predefined fraction of lines. The distribution of end-point errors is Gaussian. The degree of error is controlled by the variance of the Gaussian distribution.

- Combined errors: Here, we have introduced the four different segment errors described above in equal proportion.

The performance measure used in our sensitivity analysis is the retrieval accuracy. To compute this quantity, we perform a series of queries. The retrieval accuracy is the fraction of queries that return a correct retrieval in the top-ranked position. 


\subsection{Exact Query}

We commence with exact queries which have a precise counterpart in the database. Fig. 7 compares the performance obtained with the gated histogram, the ungated histogram, and graph-matching. The three plots show the retrieval accuracy as a function of the fraction of lines that are subjected to segmentation errors. In the case of the gated histogram, retrieval performance does not degrade until the fraction of errors exceeds 20 percent. The most destructive types of errors are line segment end-point errors and the addition of extra lines. End-point errors will have the effect of dispersing the histogram bin-contents. The addition of extra lines has the effect of saturating the histogram background, i.e., adding a uniform component to the distributions. The method is significantly less sensitive to missing lines and line-splitting. Both of these effects simply reduce the histogram contents rather than distorting its shape.

By contrast, the ungated histogram is considerably more sensitive to the addition of lines and line-splitting. The retrieval procedure displays little robustness under these two types of error. The performance under line end-point error is comparable to that of the gated histogram. However, the ungated histogram is most robust to missing line-segments. Here, retrieval performance does not degrade until the fraction of deleted lines exceeds 80 percent. By contrast, for the gated histogram, the onset of retrieval error begins when 60 percent of the lines are deleted. This is attributable to the disruption of the nearest neighbor graph by line-deletion or occlusion.

Finally, the more complicated and computationally demanding graph-matching scheme outperforms both of the histogram-based methods under each of the noise processes. However, it is some 10,000 times slower than the histogram-based comparison on a typical line pattern of some 100 lines.

\subsection{Inexact Query}

We have repeated these experiments with an inexact query. Here the query pattern is a distorted version of the target in the database. An example is furnished by the digital map which is a barrel-distorted version of the target. Fig. 8 again shows the retrieval accuracy as a function of the fraction of segmentation errors.

In the case of the gated or relational histogram, a more complex sensitivity pattern emerges. In addition to segment end-point error and missing lines, line-splitting now becomes a serious problem. This is attributable to the fact that the line-splitting introduces additional combinatorial background that swamps the query pattern. The line-patterns are least sensitive to segment end-point errors. In the case of both line-addition and line-splitting, there is an onset of errors when the fraction of segment errors is about 20 percent. However, at larger fractions of segmentation errors, the overall effect is significantly less marked than in the case of linedeletions.

When retrieval is attempted using graph matching, the following sensitivity pattern emerges. The most destructive noise process is the deletion of lines. Here the onset of retrieval errors occurs when 10 percent of lines are deleted. Line splitting results in the onset of retrieval degradation when the error rate is 20 percent. The technique is most robust to the addition of extra lines. Here, as many as 70 percent of the lines may be added clutter before any degradation in retrieval performance results.

Finally, the ungated histogram offers extremely poor recall in the case of inexact query and is almost unusable for realistic object retrieval.

\section{Discussion AND CONCLUSION}

The main contribution of this paper has been to describe a simple and compact histogram representation which combines geometrical and structural information for line-patterns. This representation can be effectively used to index into a large database according to shape similarity. The retrieval is effected by computing the Bhattacharyya distance between histograms. We show that optimal performance is obtained when the nearest neighbor graph used to gate the histogram is of order six. The method is relatively insensitive to the under-and-over segmentation of the line-patterns.

The work presented in this paper may be regarded as an important step in a more ambitious program aimed at developing a hierarchical recognition system for large image database [8]. Our next goal is to investigate the possibility of using high order statistics to measure attribute similarity. This would constitute an alternative to the histogram representation and would reduce the complexity of the search process. A second goal is to refine the rather crude index of structural similarity. In particular, we aim to derive fine grain similarity measures from the recently reported Bayesian framework for relational graph matching of Wilson and Hancock [13].

\section{REFERENCES}

[1] F. Stein and G. Medioni, "Structural Indexing: Efficient 2D Object Recognition," IEEE Trans. Pattern Analysis and Machine Intelligence, vol. 14, no. 12, pp. 1,192-1,204, Dec. 1992.

[2] E.M. Arkin, L.P. Chew, D.P. Huttenlocher, K. Kedem, and J.S.B. Mitchell, "An Efficiently Computable Metric for Comparing Polygonal Shapes," IEEE Trans. Pattern Analysis and Machine Intelligence, vol. 13, no. 3, pp. 209216, Mar. 1991.

[3] M.J. Swain, "Interactive Indexing into Image Databases," Image and Vision Storage and Retrieval, pp. 95-103, 1993.

[4] G.L. Gimelfarb and A.K. Jain, "On Retrieving Textured Images from an Image Database," Pattern Recognition, vol. 29, no. 9, pp. 1,461-1,483, 1996.

[5] R. Horaud and H. Sossa, "Polyhedral Object Recognition by Indexing," Pattern Recognition, vol. 28, no. 12, pp. 1,855-1,870, 1995.

[6] N.A. Thacker, P.A. Riocreux, and R.B. Yates, "Assessing the Completeness Properties of Pairwise Geometric Histograms," Image and Vision Computing, vol. 13, pp. 423-429, June 1995.

[7] M.S. Costa and L. Shapiro, "Scene Analysis Using Appearance-Based Models and Relational Indexing," Proc. IEEE Computer Society Int'l Symp. Computer Vision, pp. 103-108, 1995.

[8] K. Sengupta and K.L. Boyer, "Organising Large Structural Databases," IEEE Trans. Pattern Analysis and Machine Intelligence, vol. 17, no. 4, pp. 321332, 1995.

[9] Y.C. Hecker and R.M. Bolle, "On Geometric Hashing and the Generalized Hough Transform," IEEE Trans. Systems, Man, and Cybernetics, vol. 24, no. 9, pp. 1,328-1,338, Sept. 1994.

[10] F.C.D. Tsai, "Geometric Hashing with Line Features," Pattern Recognition, vol. 27, no. 3, pp. 377-389, 1994.

[11] W. Christmas, J. Kittler, and M. Petrou, "Structural Matching in Computer Vision Using Probabilistic Relaxation," IEEE Trans. Pattern Analysis and Machine Intelligence, vol. 17, pp. 749-764, 1995.

[12] W.E.L. Grimson and T. Lozano-Perez, "Localizing Overlapping Parts by Searching the Interpretation Tree," IEEE Trans. Pattern Analysis and Machine Intelligence, vol. 9, no. 4, pp. 469-482, 1987.

[13] R. Wilson and E.R. Hancock, "Structural Matching by Discrete Relaxation," IEEE Trans. Pattern Analysis and Machine Intelligence, vol. 19, no. 6, pp. 634648, June 1997.

[14] A.C. Evans, N.A. Thacker, and J.W.E. Mayhew, "The Use of Geometric Histograms for Model-Based Object Recognition," Proc. Fourth British Machine Vision Conf., pp. 429-438, Sept. 1993.

[15] M. Stricker and M. Swain, "The Capacity of Color Histogram Indexing," Proc. IEEE Conf. Computer Vision and Pattern Recognition, pp. 704-708, 1994.

[16] E.C. DiMauro, T.F. Cootes, C.J. Taylor, and A. Lanitis, “Active Shape Model Search Using Pairwise Geometric Histograms," Proc. Seventh British Machine Vision Conf., vol. 1, pp. 353-362, Sept. 1996.

[17] Y. Rubner, C. Tomasi, and L. Guibas, "A Metric for Distributions with Applications to Image Databases," Proc. ICCV'98, pp. 59-66, 1998.

[18] A.J. Bray and V. Hlavac, "Properties of Local Geometric Constraints," Proc. Second British Machine Vision Conf., pp. 95-103, Sept. 1991.

[19] B. Huet, A.D.J. Cross, and E.R. Hancock, "Graph Matching for Shape Retrieval," Advances in Neural Information Processing Systems, M.J. Kearns, S.A. Solla, and D.A. Cohn, eds., vol. 11, pp. 896-902, Cambridge, Mass.: MIT Press, June 1998. 